\title{
Checkpoint inhibitor therapy for metastatic triple-negative breast cancer
}

\author{
Arielle L. Heeke ${ }^{1}$ (D) - Antoinette R. $\operatorname{Tan}^{1}$ (D) \\ Received: 6 November 2020 / Accepted: 27 April 2021 / Published online: 8 June 2021 \\ (C) The Author(s), under exclusive licence to Springer Science+Business Media, LLC, part of Springer Nature 2021
}

\begin{abstract}
Immunotherapy has become a mainstay of cancer treatment in many malignancies, though its application in breast cancer remains limited. Of the breast cancer subtypes, triple-negative breast cancers (TNBCs) are characterized by immune activation and infiltration and more commonly express biomarkers associated with response to immunotherapy. Checkpoint inhibitor therapy has shown promising activity in metastatic TNBC. In 2019, the US FDA granted accelerated approval of atezolizumab, a programmed death-ligand 1 (PD-L1) inhibitor, in combination with nab-paclitaxel for unresectable locally advanced or metastatic PD-L1-positive TNBC, based on the results of the phase III IMpassion130 trial. In 2020, the FDA also granted accelerated approval of pembrolizumab, a PD-1 inhibitor, in combination with chemotherapy for locally recurrent unresectable and metastatic PD-L1-positive TNBC, based on results of the phase III KEYNOTE-355 trial. Additional combination strategies are being explored in the treatment of metastatic TNBC, with the goal of augmenting antitumor activity. In this review, the clinical development of checkpoint inhibitors in the treatment of metastatic TNBC will be discussed, including clinical outcomes with monotherapy and combination therapy regimens, biomarkers that may predict for benefit, and future directions in the field.
\end{abstract}

Keywords Triple-negative breast cancer $\cdot$ Metastatic $\cdot$ Immunotherapy $\cdot$ Checkpoint inhibitor $\cdot$ Review

\section{Introduction}

Immunotherapy has changed the paradigm of cancer treatment and provided a new avenue for research endeavors. Over the last decade, several immune checkpoint inhibitors have been approved by the US Food and Drug Administration (FDA) to treat cancer, with many ongoing clinical trials assessing the efficacy of next-generation immunotherapy agents, new indications, and combination therapies. Currently approved checkpoint inhibitors include programmed cell death 1 (PD1) inhibitors pembrolizumab, nivolumab, and cemiplimab; programmed death-ligand 1 (PD-L1) inhibitors atezolizumab, durvalumab, and avelumab; and the cytotoxic T lymphocyteassociated protein 4 (CTLA-4) inhibitor ipilimumab. These agents can provide a durable response in tumors characterized by tumor cell or infiltrating immune cell PD-L1 positivity, a ligand which typically binds to PD-1 on T lymphocytes to

Arielle L. Heeke

Arielle.Heeke@atriumhealth.org

Levine Cancer Institute, Atrium Health, Charlotte, NC, USA inhibit immune clearance of tumor cells. By blocking this interaction with PD-1/PD-L1 inhibitors, the immune system becomes able to recognize and eliminate tumor cells. Another mechanism for tumor immune evasion is the interaction between CTLA- 4 on T lymphocytes and the ligands CD86 and CD80 on dendritic antigen-presenting cells, which normally inhibits $\mathrm{T}$ lymphocyte response. Following exposure to the CTLA-4 inhibitor ipilimumab, priming and activation of $\mathrm{T}$ cells to recognize tumor cells becomes possible.

In breast cancer, the FDA granted accelerated approval to the anti-PD-L1 agent atezolizumab and the anti-PD-1 agent pembrolizumab, each in combination with chemotherapy, for treatment of metastatic PD-L1-positive triple-negative breast cancer (TNBC) based on progression-free survival (PFS). These regimens were evaluated in the first-line setting. These therapies are available following years of prior study of immunotherapy in TNBC (Table 1), which will be outlined in this review.

\section{Immune checkpoint inhibitors as monotherapy}

Several monotherapy phase I trials were designed after the identification of TNBC as a tumor subtype that would derive 


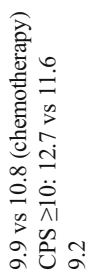

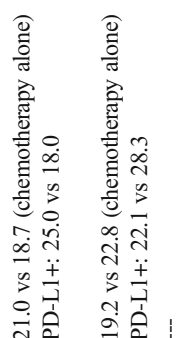

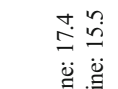

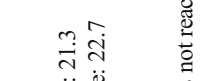

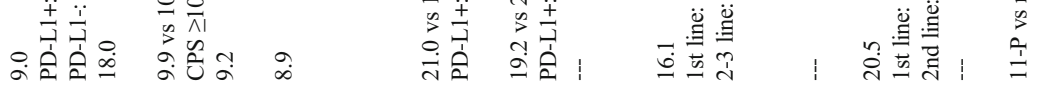

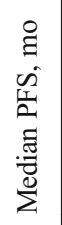

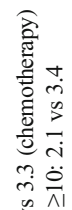

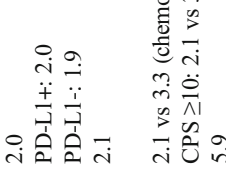

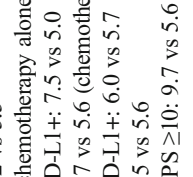

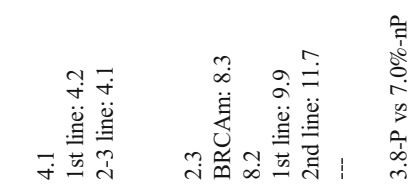

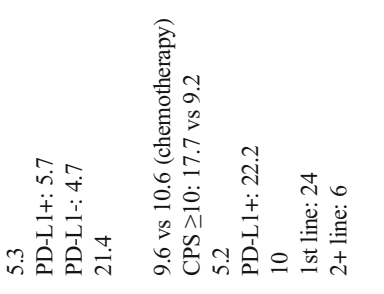

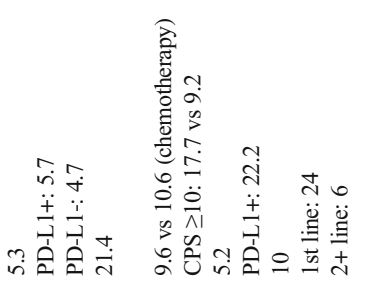

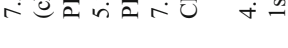

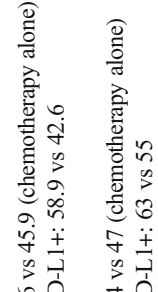

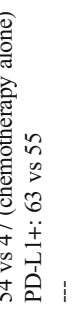

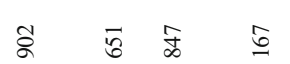

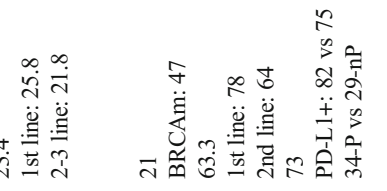

$\approx$

$\cong$ ส

तु
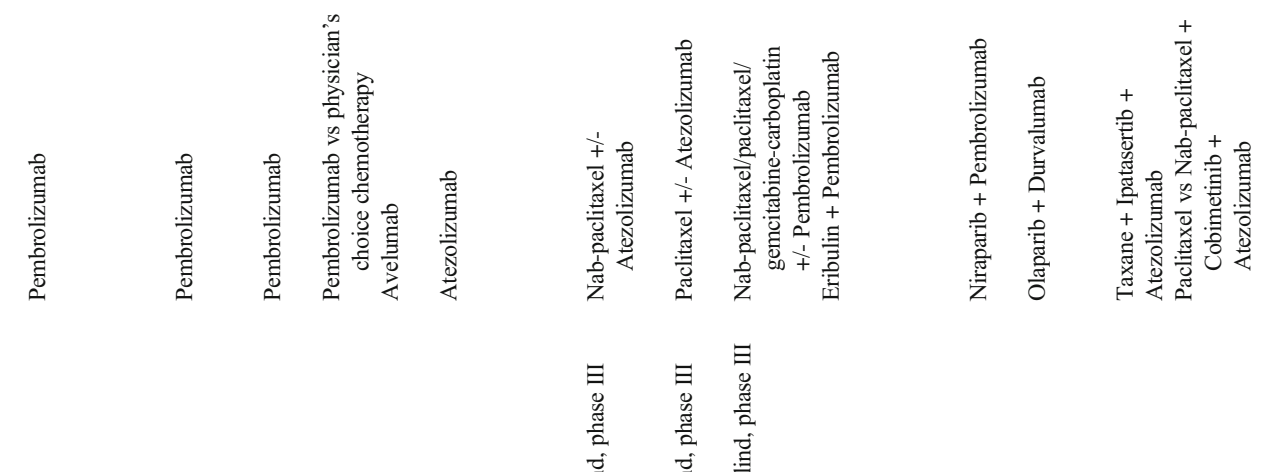
clinical benefit from immunotherapy. In the phase I KEYNOTE-012 trial, 111 patients with metastatic TNBC were screened for PD-L1 expression and 32 were enrolled to receive the PD-1 inhibitor pembrolizumab $10 \mathrm{mg} / \mathrm{kg}$ IV every 2 weeks. PD-L1 positivity was defined as staining in the stroma or in $1 \%$ or more of tumor cells by immunohistochemistry. Among the 27 patients evaluable, the overall response rate (ORR) was $18.5 \%$ with a median PFS of 1.9 months. One patient (3.7\%) had a complete response (CR), 4 (14.8\%) had a partial response (PR), and 7 (25.9\%) had stable disease with a median time to response of 17.9 weeks and median duration of response not reached $[1,2]$. With promising single-agent activity, a phase II trial was designed to evaluate the activity of pembrolizumab as monotherapy. In KEYNOTE-086, pembrolizumab $200 \mathrm{mg}$ IV every 3 weeks was evaluated in metastatic TNBC in two cohorts: (A) an unselected patient population later line and (B) PD-L1-positive tumors first-line. Flat dosing of pembrolizumab was chosen for the phase II study, following population pharmacokinetic analyses in melanoma that showed flat dosing provided exposures that maintained maximal efficacy response and tolerability as compared to the previously established $2 \mathrm{mg} / \mathrm{kg}$ dose every 3 weeks [3]. In cohort A which included 170 patients, the ORR was 5.3\% overall with a median PFS of 2.0 months and median overall survival (OS) of 9.0 months. In PD-L1-positive patients, the ORR was $5.7 \%$ [4]. In cohort B which included 84 patients, the ORR was $21.4 \%$, including 4 patients with a CR. The median PFS was 2.1 months and the median OS was 18.0 months [5]. Herein, the importance of immunotherapy administration in an earlier line setting is highlighted, as response rates in the PD-L1-positive populations differed significantly, dependent on the line of therapy.

KEYNOTE-119 was a phase III trial that enrolled 622 metastatic TNBC patients to assess pembrolizumab $200 \mathrm{mg} \mathrm{IV}$ every 3 weeks versus physician's choice chemotherapy (capecitabine, eribulin, gemcitabine, or vinorelbine) following 1-2 prior lines of treatment. PD-L1 positivity was measured by a combined positive score (CPS), which is the number of PDL1 staining cells (tumor cells, lymphocytes, macrophages) divided by the total number of viable tumor cells, multiplied by 100 . In the overall population, there were no significant differences in outcomes, including OS, ORR, and duration of response. However, in tumors classified as PD-L1 positive, a trend toward improved OS was noted, particularly as PD-L1 enrichment increased. Furthermore, for patients with tumors classified by CPS $\geq 10$, OS was 12.7 months with pembrolizumab versus 11.6 months with chemotherapy, compared to 9.9 months with pembrolizumab versus 10.8 months with chemotherapy for the overall population [6]. Given these results, pembrolizumab as monotherapy is not recommended as a second- or third-line treatment for metastatic TNBC.

In another phase Ia trial with an expansion cohort that enrolled 115 unselected patients with metastatic TNBC, treatment with the PD-L1 inhibitor atezolizumab (three different schedules employed) led to an ORR of $10.0 \%$ overall with a median duration of response of 21 months. Responses were improved in patients whose tumors were characterized by $5 \%$ or greater PD-L1 expression, with an ORR of $13.0 \%$ in PDL1-positive tumors versus $5.0 \%$ in tumors with less than $5 \%$ PD-L1 expression. The median previous lines of treatment were seven (range 0-21). For patients receiving atezolizumab in the first-line setting, the ORR was $24.0 \%$, versus $6.0 \%$ for subsequent lines. Median OS overall was 8.9 months, and $100 \%$ of responders were alive at year 2. Additionally, the median OS in the first-line setting was 17.6 months versus 7.3 months in those receiving second-line and beyond atezolizumab $(p=0.01)[7,8]$. In the phase Ib JAVELIN study, unselected patients with metastatic breast cancer were treated with avelumab, an anti-PD-L1 monoclonal antibody, 10 $\mathrm{mg} / \mathrm{kg}$ IV every 2 weeks. Of the 168 patients enrolled, 58 patients had TNBC of which $68.8 \%$ were determined to have PD-L1 -positive tumors. The ORR was just $3.0 \%$ overall, $5.2 \%$ in patients with TNBC overall, and $22.2 \%$ in TNBC tumors that were PD-L1 positive [9].

Taken together, checkpoint inhibitors as single agents in the treatment of metastatic TNBC demonstrated modest response rates overall, with more favorable responses seen in the first-line setting and in immune enriched tumors. Additionally, some patients achieved a sustained duration of response that was longer than typically achieved with chemotherapy.

\section{Immune checkpoint inhibitors combined with chemotherapy}

With modest response rates seen with immune checkpoint inhibitors as monotherapy, and with benefits only in a select group of patients, efforts have shifted toward evaluating these agents with chemotherapy. Synergistic efficacy is desired, and with combination therapies, the additional agent may help create an inflamed tumor microenvironment that stimulates responses to immunotherapy in otherwise non-responders [10].

Chemotherapy is an attractive paired agent, as following its use, high levels of antigens are released by tumor cells, suppressive immune cells are depleted, and PD-L1 is upregulated [11]. For example, when anthracyclines are employed, immunogenic cell death ensues, which activates dendritic cells and can increase the proliferation of CD8+ T cells [12]. Cyclophosphamide has also been shown to induce immunogenic cell death, suppress $\mathrm{T}$ regulatory cells, and increase the proliferation of CD8+ T cells and natural killer cells $[13,14]$. Taxanes decrease $\mathrm{T}$ regulatory and myeloid-derived suppressor cells (MDSCs) and increase tumor infiltrating lymphocyte (TIL) recruitment [15-18]. Additionally, platinum 
therapy induces immunogenic cell death and can increase major histocompatibility complex (MHC) class I complex presentation on tumor cells, which promotes $\mathrm{T}$ cell activation and downregulates MDSC function [19-21]. Gemcitabine decreases MDSCs and increases proliferation of CD8+ T cells $[22,23]$. Fortunately, these findings have translated to improved clinical outcomes when chemotherapy is combined with immunotherapy in metastatic TNBC.

Based on the results of the IMpassion130 phase III trial, atezolizumab and nab-paclitaxel is a treatment option for patients with metastatic TNBC whose tumors have PD-L1 expression. In this study, patients with untreated metastatic TNBC were randomized 1:1 to receive atezolizumab $840 \mathrm{mg}$ IV every 2 weeks plus nab-paclitaxel $100 \mathrm{mg} / \mathrm{m}^{2} \mathrm{IV}$ on days 1,8 , and 15 of a 28 -day cycle compared with placebo plus nab-paclitaxel [24]. The chemotherapy agent nabpaclitaxel was chosen to mitigate the need for pre-treatment steroids which can cause immunosuppression. All patients had to complete previous chemotherapy $\geq 12$ months before randomization. PD-L1 expression was defined as $>1 \%$ staining positive on tumor-infiltrating immune cells with the Ventana SP142 assay. In both treatment arms, $41 \%$ of patients had PD-L1-positive tumors. At the first interim analysis, at a median follow-up of 12.9 months, the median PFS was 7.2 months with atezolizumab and nab-paclitaxel, as compared with 5.5 months with placebo plus nab-paclitaxel, at a hazard ratio for progression or death of $0.80(p=0.0025)$. Among patients with PD-L1-positive tumors, median PFS was 7.5 months and 5 months, respectively, which was statistically significant $(p \leq 0.001)$. The trial met its PFS endpoint in the intent-to-treat (ITT) and PD-L1-positive group. The ORR was also significantly higher in PD-L1 -positive patients treated with atezolizumab (59\% vs $43 \%, p=0.002$ ). The median OS in the ITT was 21.3 months in the atezolizumab and nabpaclitaxel arm versus 17.6 months in the placebo plus nabpaclitaxel arm, but this was not significant $(p=0.08)$. In the PD-L1-positive subgroup, median OS was longer in the atezolizumab arm by almost 10 months ( 25.0 vs 15.5 months; HR 0.62 [95\% CI, 0.45-0.86]); however, formal statistical testing could not be conducted due to the pre-specified hierarchical statistical analysis plan requiring a statistically significant median OS in the ITT population. At the second interim analysis, again there was no statistically significant improvement observed in the median OS in the ITT population. Although an improved median OS was observed in the atezolizumab arm of the PD-L1-positive subgroup of 7 months (25 months with combination therapy vs 18 months with nab-paclitaxel and placebo), this positive result could not be formally tested [25]. In an updated survival analysis with a median follow-up of 18.8 months, the PD-L1-positive group achieved a higher median overall survival of 25.4 months with atezolizumab plus nab-paclitaxel versus 17.9 months with placebo plus nab-paclitaxel (final OS improvement of 7.5 months). The pre-specified testing hierarchy precluded a statistical assessment of this difference in the absence of a statistically significant OS benefit in the ITT population [26]. A patient with metastatic TNBC that has a tumor with PD-L1 expression $\geq 1 \%$, and previous curative chemotherapy completed $\geq 12$ months, would be a reasonable candidate for atezolizumab and nab-paclitaxel as first-line therapy given the modest PFS benefit.

In the phase III IMpassion131 trial, atezolizumab or placebo was combined with paclitaxel in patients with metastatic, previously untreated TNBC. Primary results were presented at the 2020 ESMO Congress, with no improvements in PFS or OS in the patients randomized to receive atezolizumab in addition to paclitaxel, for reasons that are not clear. These findings persisted regardless of PD-L1 positivity [27]. Subsequently, the FDA issued an alert to healthcare professionals that paclitaxel should not replace nab-paclitaxel when combined with atezolizumab for the treatment of metastatic TNBC. The phase III trial IMpassion132 is ongoing and is assessing first-line atezolizumab or placebo in metastatic TNBC patients recurring $\leq 12$ months after completing standard (neo)adjuvant anthracycline and taxane chemotherapy, combined with other chemotherapeutic agents including gemcitabine/carboplatin or capecitabine. Results are not available at the time of this publication.

KEYNOTE-355 is a phase III first-line study in patients with metastatic TNBC, which randomized eligible patients 2:1 to receive chemotherapy with or without pembrolizumab $200 \mathrm{mg}$ IV every 3 weeks. This study evaluated the efficacy of combination chemotherapy-immunotherapy with several regimens, namely nab-paclitaxel, paclitaxel, and gemcitabine/ carboplatin. Eligible patients were treatment naïve in the advanced setting and had a disease-free interval (DFI) of $\geq 6$ months from completion of adjuvant chemotherapy. A statistically significant PFS benefit was reported with the addition of pembrolizumab to chemotherapy in the 323 patients who had a CPS $\geq 10$ (9.7 months vs 5.6 months, HR 0.65, $p=0.0012$ ) [28]. While both taxane and gemcitabine/ carboplatin treatment groups were shown to benefit from the addition of pembrolizumab, the PFS benefit was more pronounced with a taxane regimen (HR 0.51 [95\% confidence interval (CI) 0.33-0.78] with taxane, HR 0.77 [95\% CI $0.53-1.11]$ with gemcitabine/carboplatin). Thirty-eight percent of patients had tumors characterized by CPS $\geq 10$ and $75 \%$ had tumors characterized by CPS $\geq 1$. The magnitude of benefit from the addition of pembrolizumab was greater for patients with CPS $\geq 10$ than CPS $\geq 1$ (CPS $\geq 1$ median PFS 7.6 months with the addition of pembrolizumab vs 5.6 months, HR $0.74, p=0.0014)$. OS data is not yet available. Following these promising results, pembrolizumab combined with chemotherapy was granted accelerated approval by the FDA in November of 2020 for the treatment of metastatic PD-L1 positive (CPS $\geq 10)$ TNBC. Of note, this trial supports the use of a 
non-taxane regimen combined with a checkpoint inhibitor that can be used in the first-line setting for metastatic TNBC and can be considered to treat patients with a DFI as short as 6 months.

When comparing IMpassion130 to KEYNOTE-355, PDL1 positivity was defined by two different tests - Ventana SP142 assay on tumor-infiltrating immune cells for IMpassion130 (the companion diagnostic for atezolizumab) and CPS via the Dako 22C3 assay for KEYNOTE-355 (the companion diagnostic for pembrolizumab). In a recent analysis of the performance of various PD-L1 assays on the biobank from IMpassion130, patients with CPS $\geq 10$ via the Dako $22 \mathrm{C} 3$ assay did not achieve as significant of an OS benefit with the addition of atezolizumab, compared to patients with PD-L1-positive tumors by SP142. Utilizing the Dako 22C3 assay to select PD-L1-positive tumors (CPS $\geq 10$ ), median OS was 22 months with atezolizumab versus 18.7 months (HR 0.77), compared to 25 months with atezolizumab versus 18 months (HR 0.71) via SP142 [29]. It is recommended that if a TNBC patient's tumor is PD-L1 positive by the Ventana SP142 assay with $a \geq 1 \%$ score, then the patient is eligible for treatment with atezolizumab plus nab-paclitaxel and if a TNBC patient's tumor is PD-L1 positive by the Dako 22C3 assay with a $\mathrm{CPS} \geq 10$, then a pembrolizumab-based regimen is appropriate.

In the phase Ib ENHANCE-1 study, pembrolizumab $200 \mathrm{mg}$ IV every 3 weeks was combined with eribulin for 167 patients with metastatic TNBC, with $44 \%$ being characterized as PD-L1 positive. Eribulin is a microtubule inhibitor that decreases TGF- $\beta$ production from cancer cells and surrounding stromal cells, a factor that causes immunosuppression and angiogenesis [30]. The combination of eribulin with a checkpoint inhibitor was anticipated to exert favorable antitumor effects, due to a combination of cytotoxic effect from the chemotherapeutic agent and restoration of tumor-specific $T$ cell response. In ENHANCE-1, clinical activity was most pronounced in patients with PD-L1-positive tumors and treated first line (ORR CPS $\geq 134.5 \%$ vs $16.1 \%$; CPS $\geq 1030.8 \%$ vs $23.4 \%$ ) [31].

Metastatic TNBC remains a challenging disease to treat without targeted therapy options. However, the combination of chemotherapy with checkpoint inhibitor therapy has shown great promise, particularly for patients with immune enriched tumors, and provides a new standard of care for first-line treatment of patients with metastatic PD-L1-positive TNBC.

\section{Immune checkpoint inhibitors in combination with targeted therapy}

In addition, a variety of targeted agents have also been evaluated in combination with immunotherapy in the treatment of metastatic TNBC, with encouraging preclinical rationale. The following combination approaches are described.

\subsection{PARP inhibitors}

A group of patients with TNBC who may particularly benefit from immunotherapy are patients with germline $B R C A 1 / 2$ mutations. Tumors characterized by a BRCA1 mutation were shown to have a higher somatic mutational load, a greater number of TILs, and increased PD-L1 and CTLA-4 expression, suggesting immune activation [32]. In another evaluation, tumors characterized by a somatic mutation in the homologous recombination DNA damage repair system (including $B R C A 1 / 2$ ) were more likely to have a higher mean tumor mutational burden (TMB), increased PD-L1 overexpression, and increased frequency of mutations in chromatin remodeling genes [33]. Additionally, measures of genomic instability (including large-scale transition scores, loss of heterozygosity, and telomeric allelic imbalance) are more commonly elevated in breast tumors harboring a BRCA1/2 mutation [34].This genomic instability, or homologous recombination deficiency (HRD), can lead to increased neoantigen presentation which may also promote an immunotherapy response [35].

Poly(ADP-ribose) polymerase (PARP) inhibitors may stimulate antigen presentation via increased $\mathrm{T}$ cell cytotoxic activity and as such are an attractive combinatorial target to induce a response to immunotherapy. PARP inhibitors have also been shown to upregulate PD-L1 expression within tumor cells [36]. In preclinical models, the combination of PARP inhibition with anti-PD-L1 therapy compared with each agent alone was shown to significantly increase therapeutic efficacy [36]. In a different model, PARP inhibitors combined with anti-CTLA-4 therapy in BRCAl-deficient ovarian tumor models were found in vitro to induce longterm survival [37]. PARP inhibitors, such as olaparib and talazoparib, as monotherapy are FDA-approved for the treatment of metastatic germline $B R C A$-mutant breast cancer based on the results from the OlympiAD and EMBRACA trials [38-41]. BRCA1 mutation carriers develop TNBC more often than $B R C A 1 / 2$ wild-type counterparts [42] and increasing evidence suggests mutations in other genes involved in the homologous recombination DNA damage repair pathway are associated with TNBC and may predict responsiveness to PARP inhibition [43, 44].

PARP inhibitors in combination with immunotherapy have been studied in several metastatic breast cancer trials. The TOPACIO trial evaluated the PARP inhibitor niraparib $200 \mathrm{mg}$ orally daily in combination with pembrolizumab $200 \mathrm{mg}$ IV every 3 weeks in patients with metastatic TNBC irrespective of $B R C A 1 / 2$ mutation status or PD-L1 expression. Of the 47 efficacy evaluable patients, ORR was $21 \%$ overall and $47 \%$ in patients with tumor $B R C A 1 / 2$ mutations [45]. This ORR is comparable to the ORR seen with single-agent PARP 
inhibitor therapy in patients with metastatic TNBC and germline BRCA1/2 mutations in the OlympiAD and EMBRACA trials, $55 \%$ and $62 \%$ respectively $[38,39]$. The addition of immunotherapy may meaningfully prolong PFS in responders, however, with a median PFS of 8.3 months for patients with a BRCA1/2 mutation enrolled in the TOPACIO trial versus 5.5 and 5.8 months for patients enrolled in the OlympiAD and EMBRACA trials, respectively. Additionally, the MEDIOLA study has evaluated the combination of the PARP inhibitor olaparib with durvalumab, an anti-PD-L1 monoclonal antibody, in patients with metastatic breast cancer and a germline $B R C A 1 / 2$ mutation. The ORR was $63.3 \%$ with the highest response rates when this combination was utilized in the first- or second-line setting (ORR $70 \%$ ) [46, 47]. Several clinical trials are ongoing to assess PARP inhibitor and immunotherapy combinations with a larger patient population and in a randomized design, including the DORA trial evaluating olaparib with durvalumab (NCT03167619) and a phase II trial of olaparib with atezolizumab (NCT02849496).

\subsection{Other targeted agents}

CDK4/6 inhibitors increase effector CD8+ T-cell infiltration, increase expression of antigen-processing and antigenpresentation genes, and suppress regulatory $\mathrm{T}$ cells - all suggesting rationale for combination with immunotherapy [48, 49]. Preclinical data have demonstrated synergy between CDK4/6 inhibition and PD-1 blockade [48]. In the clinical setting, preliminary results of an ongoing phase $1 \mathrm{~b}$ study of pembrolizumab plus abemaciclib, an oral CDK 4/6 inhibitor, in metastatic breast cancer have shown an acceptable safety profile and suggested clinical benefit, with a $14.3 \%$ ORR and a $60 \%$ rate of stable disease at 16 weeks [50].

MEK inhibitors have also been shown to increase effector $\mathrm{CD} 8+\mathrm{T}$ cell infiltration and expression of MHC class I complex presentation on TNBC cells [51], thereby increasing tumor immunogenicity. Additionally, PD-L1 expression is upregulated following MEK inhibitor exposure. Combined treatment with MEK inhibitors and PD-1 blockade has resulted in enhanced responses in vivo and in vitro in TNBC [52]. In the phase II COLET trial, the combination of the MEK inhibitor cobimetinib with atezolizumab and taxane chemotherapy in patients with treatment naive metastatic TNBC led to nonsignificant increases in PFS, ORR, and OS, with more pronounced improvements seen in PD-L1-positive and basal tumors [53].

AKT inhibition has been associated with expansion of tumor-specific lymphocytes with a memory cell phenotype [54] and AKT inhibitors are of interest for combination strategies with immunotherapy in metastatic TNBC. The combination of paclitaxel with the AKT inhibitor ipatasertib and atezolizumab in metastatic TNBC achieved an ORR of $73 \%$ in a phase Ib study, regardless of PIK3CA/AKT1/PTEN alterations or PD-L1 expression [55]. Additional trials are ongoing to assess the combination of AKT inhibitors and immunotherapy, including the IPATunity 130 trial of ipatasertib, paclitaxel, and atezolizumab (NCT03337724) and the BEGONIA trial which is evaluating the AKT inhibitor capivasertib with paclitaxel and durvalumab (NCT03742102).

\subsection{Combination with vaccines}

Breast cancer expresses many tumor-associated antigens, including HER2 and mucin 1 (MUC1), which have been the target of vaccine development [56]. Initial vaccine trials showed that vaccines could be administered safely and generate antigen-specific immune responses; however, there was minimal clinical activity. One major limitation may have been targeting shared tumor antigens, and a more effective alternative may be developing vaccines that include mutationspecific antigens that are tumor-specific. Additionally, combining vaccines with checkpoint inhibitors may increase efficacy, by increasing $\mathrm{T}$ cell activation and decreasing immunosuppression pathways. A trial evaluating a neoantigen DNA vaccine alone or with durvalumab in stage II-III TNBC is currently recruiting (NCT03199040). In the metastatic setting, an additional trial is ongoing for patients with treatment-naive metastatic TNBC who will receive gemcitabine and carboplatin, followed by nab-paclitaxel and durvalumab with or without a neoantigen vaccine (NCT03606967).

\section{Immunotherapy toxicity}

The immune checkpoint proteins, such as PD-L1, are involved in suppressing $\mathrm{T}$ cell function. When immunotherapy is employed (i.e., anti-PD-L1 therapy), tumor suppression of $T$ cell function is relieved. This immune activation may not be tumor-specific, and consequently, a patient treated with immunotherapy may experience toxicities related to immune activation. The spectrum of toxicities from immunotherapy is broad, including inflammation of the skin, colon, thyroid gland, liver, pancreas, lung, and central nervous system.

The incidence of immune-related adverse events (irAEs) is significant. In a recent meta-analysis, irAEs were appreciated in $66 \%$ of all patients treated with anti-PD-1 or anti-PD-L1 therapies, including some fatal toxicities [57]. Previous experience with irAEs has informed management guidelines, allowing for recovery for most patients and often immunotherapy rechallenge (Table 2). Some studies have suggested the development of irAEs may correlate with an improved response to therapy [58-60]. Limited data exists for the safety and efficacy of immunotherapy in immune-vulnerable populations, such as patients with pre-existing autoimmune conditions or HIV/AIDS, though generally it is favored to pursue immunotherapy in these patients if clinically appropriate [61]. 
Table 2 Immune-related adverse events

\begin{tabular}{|c|c|c|c|}
\hline irAE & Medical management & Discontinue therapy & Other interventions \\
\hline Pneumonitis & $\begin{array}{l}\text { Grade } 2+\text { : withhold treatment and administer } \\
\text { corticosteroids }(1-2 \mathrm{mg} / \mathrm{kg} \text { prednisone or } \\
\text { equivalent) followed by taper }\end{array}$ & Grade 3 or 4 , recurrent grade 2 & $\begin{array}{l}\text { Evaluate with radiographic } \\
\text { imaging if suspected }\end{array}$ \\
\hline Colitis & $\begin{array}{l}\text { Grade } 2+\text { : withhold treatment and administer } \\
\text { corticosteroids }(1-2 \mathrm{mg} / \mathrm{kg} \text { prednisone or } \\
\text { equivalent) followed by taper }\end{array}$ & Grade 4 , recurrent grade 3 & $\begin{array}{l}\text { Consider endoscopy; if } \\
\text { refractory to } \\
\text { corticosteroids consider } \\
\text { infliximab or } \\
\text { vedolizumab }\end{array}$ \\
\hline $\begin{array}{l}\text { AST or ALT elevation, } \\
\text { increased bilirubin }\end{array}$ & $\begin{array}{l}\text { Grade 2+: withhold treatment and consider } \\
\text { administration of corticosteroids }(1-2 \mathrm{mg} / \mathrm{kg} \\
\text { prednisone or equivalent) followed by taper }\end{array}$ & Grade 4 & \\
\hline Type 1 diabetes & Withhold therapy, initiate insulin & & \\
\hline Hypophysitis & $\begin{array}{l}\text { Grade 2+: withhold treatment and consider } \\
\text { administration of corticosteroids }(1-2 \mathrm{mg} / \mathrm{kg} \\
\text { prednisone or equivalent) followed by taper }\end{array}$ & Grade 3 or 4 & $\begin{array}{l}\text { Monitor for resultant } \\
\text { hypopituitarism; provide } \\
\text { hormone replacement }\end{array}$ \\
\hline Hyperthyroidism & $\begin{array}{l}\text { Grade 3+: withhold treatment or permanently } \\
\text { discontinue }\end{array}$ & & $\begin{array}{l}\text { Endocrine consultation for } \\
\text { beta-blocker or } \\
\text { thionamide }\end{array}$ \\
\hline Hypothyroidism & Grade 2+: initiate thyroid hormone replacement & & \\
\hline Myocarditis & $\begin{array}{l}\text { Grade } 1+\text { : withhold treatment, consider } \\
\text { corticosteroids based on severity }\end{array}$ & Grade 3 or 4 & \\
\hline Nephritis & $\begin{array}{l}\text { Grade } 2+\text { : withhold treatment and administer } \\
\text { corticosteroids }(1-2 \mathrm{mg} / \mathrm{kg} \text { prednisone or } \\
\text { equivalent) followed by taper }\end{array}$ & Grade 3 or 4 , consider & \\
\hline Rash & $\begin{array}{l}\text { Grade 1: topical corticosteroids } \\
\text { Grade 2: high-potency topical corticosteroids } \\
\text { Grade 3+: withhold treatment and administer } \\
\text { corticosteroids ( } 1-2 \mathrm{mg} / \mathrm{kg} \text { prednisone or } \\
\text { equivalent) followed by taper }\end{array}$ & & \\
\hline
\end{tabular}

*Additional recommendations can be obtained from NCCN: management of immune checkpoint inhibitor-related toxicities

While the risks of checkpoint inhibitor therapy should be considered for each patient, the benefits of this therapy can be paramount. Moreover, durable responses can be achieved in patients with metastatic TNBC. Patients with immuneenriched advanced TNBC should be considered for immunotherapies, with close monitoring and multi-disciplinary management of irAEs should they arise.

\section{Biomarkers of response to immunotherapy}

It has been well-established that certain cancers, such as lung cancer and melanoma, respond to immunotherapy treatment algorithms, whereas other cancers do not achieve a robust response. Extensive work has been carried out to determine why certain cancers respond, and which patients within traditionally non-responsive cancers will achieve a response. An active and enriched tumor immune microenvironment is important for predicting response to immunotherapy, with higher rates of response in tumors with elevated TILs, PD-L1 positivity, and an increased density of tumor-infiltrating CD8+ T cells [62]. Such tumors have been identified as "inflamed" or "hot" tumors.

Conversely, tumors with low levels of TILs, PD-L1 positivity, and CD8+ T cells are considered "non-inflamed" or "cold," with a lower likelihood of response to immunotherapy. Breast cancer tumors more commonly fall in this category. In the initial phase I studies of immunotherapy as monotherapy, triple-negative breast tumors of the breast cancer subtypes were identified as being more likely to achieve a response. Subsequently, it has been shown that TNBC patients have tumors that are "hotter" than other breast cancer subtypes, with higher expression levels of PD-L1 [63], increased genomic instability and higher TMB leading to increased neoantigen presentation [64-66], and higher levels of TILs [67].

Exploratory analysis has confirmed these inflammatory markers can identify which breast tumors will be more likely to respond to immunotherapy. Biomarker evaluation as part of the IMpassion 130 trial (evaluating nab-paclitaxel with or without atezolizumab first-line in metastatic TNBC) determined PD-L1 expression on immune cells by the VENTANA SP142 IHC assay to be highly predictive of response, with significant improvements seen in both PFS and 
OS in the PD-L1-positive patient cohort [24]. TILs, and specifically a higher density of CD8+ cytotoxic TILs, are also positively correlated with immunotherapy response. KEYNOTE-173 (a phase Ib trial evaluating neoadjuvant chemotherapy with or without pembrolizumab in early TNBC) showed that a higher combined score evaluating levels of stromal TILs and PD-L1 expression was significantly associated with higher pathologic complete response and overall response rates in patients with early-stage TNBC [68]. Furthermore, in a retrospective tissue evaluation of earlystage breast tumors, a high number of $\mathrm{CD} 8+\mathrm{T}$ cells with features of tissue-resident memory $\mathrm{T}\left(\mathrm{T}_{\mathrm{RM}}\right)$ cell differentiation were significantly associated with improved survival in TNBC [69]. In the metastatic setting, a higher density of CD8+ TILs was associated with increased PFS and OS in the IMpassion130 trial [70]. Stromal TILs were associated with PFS benefit only.

In addition to an assessment for levels of TILs, PD-L1 expression, and TMB, gene signatures may also identify breast tumors primed for an immune response. In a comprehensive genomic analysis of TNBC, four distinct gene signatures were identified-luminal androgen receptor (LAR), mesenchymal (MES), basal-like immunosuppressed (BLIS), and basal-like immune-activated (BLIA) - of which the BLIA subtype is considered "inflamed" and "hot" and primed for response to immunotherapy. In the BLIA subtype, tumors tend to upregulate genes controlling B cell, $\mathrm{T}$ cell, and natural killer cell functions, whereas in the BLIS subtype, tumors tend to decrease expression of molecules controlling antigen presentation, immune cell differentiation, and innate and adaptive immune cell communication [71]. While hypothesis-generating, this subclassification has not been correlated with treatment response, but has been associated with outcome, with the best prognosis achieved with the BLIA subtype and worst disease-free survival in the BLIS subtype. In another evaluation where breast cancer tumors were classified into one of four groups based on their immune-related gene signatures, patients with tumors classified as highly immunogenic (with upregulation of immunoregulatory transcripts including PDL1, PD-1, FOXP3, IDO1, and CTLA-4) achieved prolonged survival [72]. Again, this evaluation did not include a correlation with immunotherapy treatment response.

Immunotherapy biomarker expression is influenced by tissue source. Moreover, immune-enriched tumors are more commonly identified from the primary breast tumor, compared to a metastatic tumor. In a paired analysis of 76 TNBC tumors for TIL count and PD-L1 positivity (using the E1L3N XP rabbit monoclonal antibody, with positivity defined as $\geq 1 \%$ positive tumor, or stromal cells), both biomarkers were significantly decreased among metastatic tumors compared to primary tumors [73]. Of note in this study, tissue samples derived from tissue microarray compared to the whole section (whether metastatic or primary) had overall lower biomarker expression as well. In another study of the Foundation Medicine clinical database including 340 TNBC cases, PD-L1 positivity (as defined by the SP142 assay [ $\geq 1 \%$ immune cells stained positive]) was significantly more common among primary tumors compared to metastatic tumors $(63.7 \%$ vs $42.4 \%, p<0.0001)$ [74]. In the majority of the clinical trials referenced in Table 1, tissue was allowable from both the primary breast or a metastatic site, with fresh tissue and a metastatic site preferred, though archived and/or primary breast tissue allowable. Limited analyses exist from these trials regarding source of tissue and biomarker expression. In a post hoc analysis of the IMpassion130 trial that assessed atezolizumab with nab-paclitaxel in advanced TNBC, PDL1 positivity was more common if testing was performed on breast tissue (comprising $62 \%$ of available tissue) compared to a metastatic site, $44 \%$ versus $36 \%$ [29]. Given these data, PDL1 testing ideally can be performed on a metastatic tumor and if not available, then it is reasonable to test on primary breast tissue.

\section{Concluding remarks}

With currently available and evolving data, checkpoint inhibitor therapy should be offered to metastatic TNBC patients with an identified biomarker predictive of response (i.e., PDL1 overexpression or an elevated CPS). Timing is important as patients should be evaluated for biomarkers of response at time of initial diagnosis, with immunotherapy utilized in the first-line setting when possible. Several studies have highlighted the best response to immunotherapy, and a durable response is achieved when therapy is used in the first-line setting. It is not clear if one checkpoint inhibitor is better than another, nor which biomarker assay most reliably predicts for response, and additional work is needed in this area to help select the best treatment for an individual patient. Many exciting combination approaches are being evaluated, and patients should consider clinical trial opportunities when available.

\section{Declarations}

Conflict of interest Arielle Heeke: Honoraria from Merck and Pfizer.

Antoinette Tan: Research funding to the institution for clinical trial support and honoraria from Merck and Genentech/Roche.

\section{References}

1. Nanda R, Chow LQ, Dees EC, Berger R, Gupta S, Geva R, et al. (2016). Pembrolizumab in patients with advanced triple-negative breast cancer: Phase Ib KEYNOTE-012 Study. Journal of Clinical Oncology, 34(21), 2460-2467. 
2. Nanda R, Specht J, Dees C, Berger R, Gupta S, Geva R, et al. (2016). KEYNOTE-012: Long-lasting responses in a phase $\mathrm{Ib}$ study of pembrolizumab for metastatic triple negative breast cancer. San Antonio Breast Cancer Symposium 2016. Cancer Research, 77(suppl 4): P6-10-03.

3. Freshwater T, Kondic A, Ahamadi M, Li CH, de Greef R, de Alwis D, et al. (2017). Evaluation of dosing strategy for pembrolizumab for oncology indications. Journal for Immunotherapy of Cancer, 5 , 43.

4. Adams S, Schmid P, Rugo HS, Winer EP, Loirat D, Awada A, et al. (2019). Pembrolizumab monotherapy for previously treated metastatic triple-negative breast cancer: Cohort A of the phase II KEYNOTE-086 study. Annals of Oncology, 30(3), 397-404.

5. Adams S, Loi S, Toppmeyer D, Cescon DW, De Laurentiis M, Nanda R, et al. (2019). Pembrolizumab monotherapy for previously untreated, PD-L1-positive, metastatic triple-negative breast cancer: Cohort B of the phase II KEYNOTE-086 study. Annals of Oncology, 30(3), 405-411.

6. Winer EP, Lipatov O, Im SA, Goncalves A, Munoz-Couselo E, Lee KS, et al. (2021). Pembrolizumab versus investigator-choice chemotherapy for metastatic triple-negative breast cancer (KEYNOTE-119): A randomised, open-label, phase 3 trial. The Lancet Oncology, 22(4), 499-511.

7. Schmid P, Cruz C, Braiteh FS, Eder JP, Tolaney SM, Kuter I, et al. (2017). Atezolizumab in metastatic TNBC (mTNBC): Long-term clinical outcomes and biomarker analyses. AACR Annual Meeting 2017. Clinical Cancer Research, 77(suppl_13), 2986.

8. Emens LA, Cruz C, Eder JP, Braiteh F, Chung C, Tolaney SM, et al. (2019). Long-term clinical outcomes and biomarker analyses of atezolizumab therapy for patients with metastatic triple-negative breast cancer: A phase 1 study. JAMA Oncology, 5(1), 74-82.

9. Dirix LY, Takacs I, Jerusalem G, Nikolinakos P, Arkenau HT, Forero-Torres A, et al. (2018). Avelumab, an anti-PD-L1 antibody, in patients with locally advanced or metastatic breast cancer: a phase 1b JAVELIN Solid Tumor study. Breast Cancer Research and Treatment, 167(3), 671-686.

10. Emens LA, \& Middleton G. (2015). The interplay of immunotherapy and chemotherapy: Harnessing potential synergies. Cancer Immunology Research, 3(5), 436-443.

11. Fridman WH, Zitvogel L, Sautes-Fridman C, \& Kroemer G. (2017). The immune contexture in cancer prognosis and treatment. Nature Reviews Clinical Oncology, 14(12), 717-734.

12. Galluzzi L, Buque A, Kepp O, Zitvogel L, \& Kroemer G. (2017). Immunogenic cell death in cancer and infectious disease. Nature Reviews Immunology, 17(2), 97-111.

13. Ghiringhelli F, Menard C, Puig PE, Ladoire S, Roux S, Martin F, et al. (2007). Metronomic cyclophosphamide regimen selectively depletes CD4+CD25+ regulatory $\mathrm{T}$ cells and restores $\mathrm{T}$ and NK effector functions in end stage cancer patients. Cancer Immunology, Immunotherapy, 56(5), 641-648.

14. Kwa M, Li X, Novik Y, Oratz R, Jhaveri K, Wu J, et al. (2018). Serial immunological parameters in a phase II trial of exemestane and low-dose oral cyclophosphamide in advanced hormone receptor-positive breast cancer. Breast Cancer Research and Treatment, 168(1), 57-67.

15. Demaria S, Volm MD, Shapiro RL, Yee HT, Oratz R, Formenti SC, et al. (2001). Development of tumor-infiltrating lymphocytes in breast cancer after neoadjuvant paclitaxel chemotherapy. Clinical Cancer Research, 7(10), 3025-3030.

16. Kodumudi KN, Woan K, Gilvary DL, Sahakian E, Wei S, \& Djeu J Y. (2010). A novel chemoimmunomodulating property of docetaxel: suppression of myeloid-derived suppressor cells in tumor bearers. Clinical Cancer Research, 16(18), 4583-4594.

17. Roselli M, Cereda V, di Bari MG, Formica V, Spila A, Jochems C, et al. (2013). Effects of conventional therapeutic interventions on the number and function of regulatory T cells. Oncoimmunology, 2(10), e27025.

18. Li JY, Duan XF, Wang LP, Xu YJ, Huang L, Zhang TF, et al. (2014). Selective depletion of regulatory $T$ cell subsets by docetaxel treatment in patients with nonsmall cell lung cancer. Journal of Immunology Research, 2014, 286170.

19. Jackaman C, Majewski D, Fox SA, Nowak AK, \& Nelson DJ. (2012). Chemotherapy broadens the range of tumor antigens seen by cytotoxic CD8(+) T cells in vivo. Cancer Immunology, Immunotherapy, 61(12), 2343-2356.

20. Nio Y, Hirahara N, Minari Y, Iguchi C, Yamasawa K, Toga T, et al. (2000). Induction of tumor-specific antitumor immunity after chemotherapy with cisplatin in mice bearing MOPC-104E plasmacytoma by modulation of MHC expression on tumor surface. Anticancer Research, 20(5A), 3293-3299.

21. Huang X, Cui S, \& Shu Y. (2016). Cisplatin selectively downregulated the frequency and immunoinhibitory function of myeloidderived suppressor cells in a murine B16 melanoma model. Immunologic Research, 64(1), 160-170.

22. Homma Y, Taniguchi K, Nakazawa M, Matsuyama R, Mori R, Takeda K, et al. (2014). Changes in the immune cell population and cell proliferation in peripheral blood after gemcitabine-based chemotherapy for pancreatic cancer. Clinical \& Translational Oncology, 16(3), 330-335.

23. Vincent J, Mignot G, Chalmin F, Ladoire S, Bruchard M, Chevriaux A, et al. (2010). 5-Fluorouracil selectively kills tumorassociated myeloid-derived suppressor cells resulting in enhanced T cell-dependent antitumor immunity. Cancer Research, 70(8), 3052-3061.

24. Schmid P, Adams S, Rugo HS, Schneeweiss A, Barrios CH, Iwata $\mathrm{H}$, et al. (2018). Atezolizumab and nab-paclitaxel in advanced triple-negative breast cancer. The New England Journal of Medicine, 379(22), 2108-2121.

25. Schmid P, Rugo HS, Adams S, Schneeweiss A, Barrios CH, Iwata $\mathrm{H}$, et al. (2020). Atezolizumab plus nab-paclitaxel as first-line treatment for unresectable, locally advanced or metastatic triplenegative breast cancer (IMpassion130): updated efficacy results from a randomised, double-blind, placebo-controlled, phase 3 trial. The Lancet Oncology, 21(1), 44-59.

26. Emens LA, Adams S, Barrios CH, Dieras VC, Iwata H, Loi S, et al. (2020). IMpassion130: Final OS analysis from the pivotal phase III study of atezolizumab + nab-paclitaxel vs placebo + nab-paclitaxel in previously untreated locally advanced or metastatic triplenegative breast cancer. ESMO 2020 Virtual Congress. Annals of Oncology, 31(suppl_4), S1142-SS215.

27. Miles DW, Gligorov J, Andre F, Cameron D, Schneeweiss A, Barrios CH, et al. (2020). Primary results from IMpassion131, a double-blind placebo-controlled randomised phase III trial of firstline paclitaxel (PAC) +/- atezolizumab (atezo) for unresectable locally advanced/metastatic triple-negative breast cancer (mTNBC). ESMO 2020 Virtual Congress. Annals of Oncology, (suppl_4), S1142-SS215.

28. Cortes J, Cescon DW, Rugo HS, Nowecki Z, Im SA, Yusof MM, et al. (2020). Pembrolizumab plus chemotherapy versus placebo plus chemotherapy for previously untreated locally recurrent inoperable or metastatic triple-negative breast cancer (KEYNOTE-355): A randomised, placebo-controlled, double-blind, phase 3 clinical trial. Lancet, 396(10265), 1817-1828.

29. Rugo HS, Loi S, Adams S, Schmid P, Schneeweiss A, Barrios CH, et al. (2019). Performance of PD-L1 immunohistochemistry (IHC) assays in unresectable locally advanced or metastatic triple-negative breast cancer (mTNBC): Post-hoc analysis of IMpassion 130. ESMO 2019 Congress. Annals of Oncology, 30(suppl_5), v851v934.

30. Ueda S, Saeki T, Takeuchi H, Shigekawa T, Yamane T, Kuji I, et al. (2016). In vivo imaging of eribulin-induced reoxygenation in 
advanced breast cancer patients: A comparison to bevacizumab. British Journal of Cancer, 114(11), 1212-1218.

31. Tolaney SM, Kalinsky K, Kaklamani VG, D'Adamo DR, Aktan G, Tsai ML, et al. (2021). Eribulin plus pembrolizumab in patients with metastatic triple-negative breast cancer (ENHANCE 1): A phase ib/ii study. Clinical Cancer Research, online ahead of print.

32. Nolan ESP, Policheni AN, Darcy PK, Vaillant F, Mintoff CP, Dushyanthen S, et al. (2017). Combined immune checkpoint blockade as a therapeutic strategy for BRCA1-mutated breast cancer. Science Translational Medicine, 9(393), eaal4922.

33. Heeke AL, Xiu J, Elliott A, Korn WM, Lynce F, Pohlmann PR, et al. (2020). Actionable coalterations in breast tumors with pathogenic mutations in the homologous recombination DNA damage repair pathway. Breast Cancer Research and Treatment, 184(2): 265-275.

34. Timms KM, Abkevich V, Hughes E, Neff C, Reid J, Morris B, et al. (2014). Association of BRCA $1 / 2$ defects with genomic scores predictive of DNA damage repair deficiency among breast cancer subtypes. Breast Cancer Research, 16(6), 475.

35. Mardis ER. (2019). Neoantigens and genome instability: Impact on immunogenomic phenotypes and immunotherapy response. Genome Medicine, 11(1), 71.

36. Jiao S, Xia W, Yamaguchi H, Wei Y, Chen MK, Hsu JM, et al. (2017). PARP inhibitor upregulates PD-L1 expression and enhances cancer-associated immunosuppression. Clinical Cancer Research, 23(14), 3711-3720.

37. Higuchi T, Flies DB, Marjon NA, Mantia-Smaldone G, Ronner L, Gimotty PA, et al. (2015). CTLA-4 blockade synergizes therapeutically with PARP inhibition in BRCA1-deficient ovarian cancer. Cancer Immunology Research, 3(11), 1257-1268.

38. Eiermann W, Rugo HS, Diab S, Ettl J, Hurvitz SA, Goncalves A, et al. (2018). Analysis of germline BRCA1/2 mutated (gBRCAmut) hormone receptor-positive $(\mathrm{HR}+)$ and triple negative breast cancer (TNBC) treated with talazoparib (TALA). ASCO Annual Meeting 2018. Journal of Clinical Oncology, 36(suppl_15), abstract 1070.

39. Senkus-Konefka E, Domchek SM, Im SA, Xu B, Amrstrong AC, Masuda N, et al. Subgroup analysis of olaparib monotherapy versus chemotherapy by hormone receptor and BRCA mutation status in patients with HER2-negative metastatic breast cancer and a germline BRCA mutation: OlympiAD. European Breast Cancer Conference 2018. European Journal of Cancer, 92(suppl_3), S19-S20.

40. Litton JK, Rugo HS, Ettl J, Hurvitz SA, Goncalves A, Lee KH, et al. (2018). Talazoparib in patients with advanced breast cancer and a germline BRCA mutation. The New England Journal of Medicine, 379(8), 753-763.

41. Robson M, Im SA, Senkus E, Xu B, Domchek SM, Masuda N, et al. (2017). Olaparib for metastatic breast cancer in patients with a germline BRCA mutation. The New England Journal of Medicine, 377(6), 523-533.

42. Lakhani SR, Van De Vijver MJ, Jacquemier J, Anderson TJ, Osin PP, McGuffog L, et al. (2002). The pathology of familial breast cancer: predictive value of immunohistochemical markers estrogen receptor, progesterone receptor, HER-2, and p53 in patients with mutations in BRCA1 and BRCA2. Journal of Clinical Oncology, 20(9), 2310-2318.

43. Tung NM, Robson ME, Ventz S, Santa-Maria CA, Marcom PK, Nanda R, et al. (2020). TBCRC 048: A phase II study of olaparib monotherapy in metastatic breast cancer patients with germline or somatic mutations in DNA damage response (DDR) pathway genes (Olaparib Expanded). ASCO Annual Meeting 2020. Journal of Clinical Oncology, 38(suppl_15), abstract 1002.

44. Domagala P, Jakubowska A, Jaworska-Bieniek K, Kaczmarek K, Durda K, Kurlapska A, et al. (2015). Prevalence of Germline mutations in genes engaged in DNA damage repair by homologous recombination in patients with triple-negative and hereditary nontriple-negative breast cancers. PLoS One, 10(6), e0130393.

45. Vinayak S, Tolaney SM, Schwartzberg L, Mita M, McCann G, Tan AR, et al. (2019). Open-label clinical trial of niraparib combined with pembrolizumab for treatment of advanced or metastatic triplenegative breast cancer. JAMA Oncology, 5(8), 1132-1140.

46. Domchek SP, Postel-Vinay S, Im SA, Park YH, Delord JP, Italiano A, et al. (2019). Phase II study of olaparib (O) and durvalumab (D) (MEDIOLA): Updated results in patients (pts) with germline BRCA-mutated (gBRCAm) metastatic breast cancer (MBC). ESMO 2019 Congress. Annals of Oncology, 30(suppl_5), v475v532.

47. Domchek SM, Postel-Vinay S, Im SA, Park YH, Delord JP, Italiano A, et al. (2020). Olaparib and durvalumab in patients with germline BRCA-mutated metastatic breast cancer (MEDIOLA): an open-label, multicentre, phase $1 / 2$, basket study. The Lancet Oncology, 21, 1155-1164.

48. Deng J, Wang ES, Jenkins RW, Li S, Dries R, Yates K, et al. (2018). CDK4/6 Inhibition augments antitumor immunity by enhancing t-cell activation. Cancer Discovery, 8(2), 216-233.

49. Goel S, DeCristo MJ, Watt AC, BrinJones H, Sceneay J, Li BB, et al. (2017). CDK4/6 inhibition triggers anti-tumour immunity. Nature, 548(7668), 471-475.

50. Tolaney SM, Kabos P, Dickler MN, Gianni L, Jansen V, Lu Yi, et al. (2018) Updated efficacy, safety, \& PD-L1 status of patients with HR+, HER2- metastatic breast cancer administered abemaciclib plus pembrolizumab. ASCO Annual Meeting 2018. Journal of Clinical Oncology, 36(suppl_15), abstract 1059.

51. Ebert PJR, Cheung J, Yang Y, McNamara E, Hong R, Moskalenko $\mathrm{M}$, et al. (2016). MAP kinase inhibition promotes T cell and antitumor activity in combination with PD-L1 checkpoint blockade. Immunity, 44(3), 609-621.

52. Loi S, Dushyanthen S, Beavis PA, Salgado R, Denkert C, Savas P, et al. (2016). RAS/MAPK activation is associated with reduced tumor-infiltrating lymphocytes in triple-negative breast cancer: therapeutic cooperation between MEK and PD-1/PD-L1 immune checkpoint inhibitors. Clinical Cancer Research, 22(6), 14991509.

53. Brufsky A, Kim SB, Zvirbule Z, Eniu A, Mebis J, Sohn JH, et al. (2021). A phase II randomized trial of cobimetinib plus chemotherapy, with or without atezolizumab, as first-line treatment for patients with locally advanced or metastatic triple-negative breast cancer (COLET): primary analysis. Annals of Oncology, 32(5), 652660.

54. Crompton JG, Sukumar M, Roychoudhuri R, Clever D, Gros A, Eil RL, et al. (2015). Akt inhibition enhances expansion of potent tumor-specific lymphocytes with memory cell characteristics. Cancer Research, 75(2), 296-305.

55. Schmid P, Loirat $\mathrm{D}$, Savas $\mathrm{P}$, Espinosa E, Boni V, Italiano A, et al. (2019). Phase Ib study evaluating a triplet combination of ipatasertib (IPAT), atezolizumab (atezo), and paclitaxel (PAC) or nab-PAC as first-line (1L) therapy for locally advanced/metastatic triple-negative breast cancer (TNBC). AACR Annual Meeting 2019. Cancer Research, 79(suppl_13), abstract CT049.

56. Emens LA. (2012). Breast cancer immunobiology driving immunotherapy: Vaccines and immune checkpoint blockade. Expert Review of Anticancer Therapy, 12(12), 1597-1611.

57. Wang Y, Zhou S, Yang F, Qi X, Wang X, Guan X, et al. (2019). Treatment-related adverse events of PD-1 and PD-L1 inhibitors in clinical trials: A systematic review and meta-analysis. JAMA Oncology, 5(7), 1008-1019.

58. Maher VE, Fernandes LL, Weinstock C, Tang S, Agarwal S, Brave M, et al. (2019). Analysis of the association between adverse events and outcome in patients receiving a programmed death protein 1 or programmed death ligand 1 antibody. Journal of Clinical Oncology, 37(30), 2730-2737. 
59. Schweizer C, Schubert P, Rutzner S, Eckstein M, Haderlein M, Lettmaier S, et al. (2020). Prospective evaluation of the prognostic value of immune-related adverse events in patients with nonmelanoma solid tumour treated with PD-1/PD-L1 inhibitors alone and in combination with radiotherapy. European Journal of Cancer, 140, 55-62.

60. Akamatsu H, Murakami E, Oyanagi J, Shibaki R, Kaki T, Takase E, et al. (2020). Immune-related adverse events by immune checkpoint inhibitors significantly predict durable efficacy even in responders with advanced non-small cell lung cancer. Oncologist, 25(4), e679-ee83.

61. Abdel-Wahab N, Shah M, Lopez-Olivo MA, \& Suarez-Almazor ME. (2018). Use of immune checkpoint inhibitors in the treatment of patients with cancer and preexisting autoimmune disease. Annals of Internal Medicine, 169(2), 133-134.

62. Woo SR, Corrales L, \& Gajewski TF. (2015). The STING pathway and the $\mathrm{T}$ cell-inflamed tumor microenvironment. Trends in Immunology, 36(4), 250-256.

63. Mittendorf EA, Philips AV, Meric-Bernstam F, Qiao N, Wu Y, Harrington S, et al. (2014). PD-L1 expression in triple-negative breast cancer. Cancer Immunology Research, 2(4), 361-370.

64. Budczies J, Bockmayr M, Denkert C, Klauschen F, Lennerz JK, Gyorffy B, et al. (2015). Classical pathology and mutational load of breast cancer - Integration of two worlds. The Journal of Pathology. Clinical Research, 1(4), 225-238.

65. Banerji S, Cibulskis K, Rangel-Escareno C, Brown KK, Carter SL, Frederick AM, et al. (2012). Sequence analysis of mutations and translocations across breast cancer subtypes. Nature, 486(7403), 405-409.

66. Lynce L, Xiu J, Obeid E, Tan AR, Gatalica Z, Isaacs C, et al. (2017). Tumor mutational load in gynecological and breast cancer. ASCO-SITC Clinical Immuno-Oncology Symposium 2017. Journal of Clinical Oncology, 35(suppl 7S), abstract 44.

67. Loi S, Michiels S, Salgado R, Sirtaine N, Jose V, Fumagalli D, et al. (2014). Tumor infiltrating lymphocytes are prognostic in triple negative breast cancer and predictive for trastuzumab benefit in early breast cancer: Results from the FinHER trial. Annals of Oncology, 25(8), 1544-1550.

68. Loi S, Schmid P, Aktan G, Karantza V, \& Salgado R. Relationship between tumor infiltrating lymphocytes (TILs) and response to pembrolizumab (Pembro) +chemotherapy (CT) as neoadjuvant treatment (NAT) for triple-negative breast cancer (TNBC): phase Ib KEYNOTE-173 trial. ESMO Breast Cancer 2019. Annals of Oncology, 30(suppl_3), iii1-iii26 abstract 573.

69. Savas P, Virassamy B, Ye C, Salim A, Mintoff CP, Caramia F, et al. (2018). Single-cell profiling of breast cancer T cells reveals a tissueresident memory subset associated with improved prognosis. Nature Medicine, 24(7), 986-993.

70. Adams S, Diamond JR, Hamilton E, Pohlmann PR, Tolaney SM, Chang CW, et al. (2019). Atezolizumab plus nab-paclitaxel in the treatment of metastatic triple-negative breast cancer with 2-year survival follow-up: A phase $1 \mathrm{~b}$ clinical trial. JAMA Oncology, 5(3), 334-342.

71. Burstein MD, Tsimelzon A, Poage GM, Covington KR, Contreras A, Fuqua SA, et al. (2015). Comprehensive genomic analysis identifies novel subtypes and targets of triple-negative breast cancer. Clinical Cancer Research, 21(7), 1688-1698.

72. Hendrickx W, Simeone I, Anjum S, Mokrab Y, Bertucci F, Finetti $P$, et al. (2017). Identification of genetic determinants of breast cancer immune phenotypes by integrative genome-scale analysis. Oncoimmunology, 6(2), e1253654.

73. Szekely B, Bossuyt V, Li X, Wali VB, Patwardhan GA, Frederick $\mathrm{C}$, et al. (2018). Immunological differences between primary and metastatic breast cancer. Annals of Oncology, 29(11), 2232-2239.

74. Rozenblit M, Huang R, Danziger N, Hegde P, Alexander B, Ramkissoon S, et al. (2020). Comparison of PD-L1 protein expression between primary tumors and metastatic lesions in triple negative breast cancers. Journal for Immunotherapy of Cancer, 8(2), e001558.

Publisher's note Springer Nature remains neutral with regard to jurisdictional claims in published maps and institutional affiliations. 